\title{
A Loeb-measure Approach to Theorems by Prohorov, Sazonov, and Gross
}

by

Tom L. Lindstrøm, Oslo.

\section{Introduction.}

It has been clear for some time now that the Loeb-measure of nonstandard analysis [11] may be useful in the construction of different kinds of limit measures. Indeed, Anderson's nonstandard construction of a Brownian motion [1], may be regarded as a direct construction of a weak limit measure (compare Billingsley [4]). Work on weak convergence from a nonstandard point of view have been carried on by Anderson and Rashid [3], and Loeb [12]. In another direction, Helms and Loeb [7], Hurd [9], and Helms [6] have used the Loeb-measure to obtain limit equilibrium measures in statistzcal mechanics。

In this paper we shall work with other - but related concepts of limit measures, and we hope to show the efficiency of the Loeb-measure approach by giving simple proofs of three classical theorems.

The first of these theorems is due to Prohorov [14]: Given a projective system $\left\langle x_{i}, \tau_{i}, \pi_{i j}\right\rangle_{i, j} \in I$ of Hausdorff spaces endowed with a cylindrical measure $\left\{\mu_{i}\right\}_{i \in I}$ of Radon measures on the $X_{i}$, it gives a characterization of when there is a limit Radon measure on the projective limit. The idea of the proof is 
to construct the limit measure from the Loeb-measure of $\mu_{i}$ for an infinite $i \epsilon^{*} I$, using a standard part map $\theta_{i}: X_{i} \rightarrow X_{0}$ The second theorem is due to Sazonov [16]; it characterizes the functions that are Fourier-transforms of probability measures on Hilbert spaces. The idea of the proof is that even when the measure does not exist on the Hilbert space H, measures exist on the byperfinite dimensional subspaces of ${ }^{*} \mathrm{H}$ and we can perform the necessary calculations on these spaces.

The last theorem is by Gross [5] and is concerned with the extension of cylindrical measures on Hilbert spaces to measures on Banach spaces where the Hilbert space is densely embedded. Again the idea is the same; we use the Loeb-measures on hyperfinite dimensional subspaces and the standard part map.

Throughout this paper we shall work with polysaturated models for nonstandard analysis (see Stroyan and Iuxemburg, [18])。 For an introduction to the theory of the Loeb-measure and some of its applications, the reader should consult Loeb [13].

I would like to thank Jens Erik Fenstad and Bent Birkeland for helpful discussions and encouragement during the work on this paper.

II. Measures on inductive limits: Prohorov's theorem.

Let $\left\langle X_{i}, \tau_{i}, \pi_{i j}\right\rangle_{i, j \in I}$ be a projective system of Hausdorff spaces; i。e. I is a directed set; for each $i \in I,\left\langle X_{i}, \tau_{j}\right\rangle$ is a Hausdorff space; and for all $i, j, k \in I, \quad i \leq j \leq k, \quad \pi_{i j}: X_{j} \rightarrow X_{i}$, $\pi_{j k}: X_{k} \rightarrow X_{j}$, and $\pi_{i k}: X_{k} \rightarrow X_{i}$ are continuous, surjective maps satisfying $\pi_{i k}=\pi_{i j}{ }^{\circ} \pi_{j k}$. By the projective limit of such 
a system, we shall mean the Hausdorff space $\langle X, \tau\rangle$, where $X$ consists of all elements $x \in \Pi X_{i}$ such that for all $i, j \in I, i \leq j$, $x_{i}=\pi_{i j}\left(x_{j}\right)$; and where $\tau$ is the weakest topology making a.II the maps $\pi_{i}: X \rightarrow X_{i}$-defined by $\pi_{i}(x)=x_{i}$-continuous. Obviously $\pi_{i}=\pi_{i j}{ }^{\circ} \pi_{j}$ for $i \leq j$. The set $X$ may be empty even when all the $X_{i}$ are non-empty; and the $\pi_{i}$ need not be surjective.

We shall denote the ${ }^{*}$-version ${ }^{*}\left\{\left\langle X_{i}, \tau_{i}, \pi_{i j}\right\rangle_{i, j \in I}\right\}$ by $\left\langle\tilde{X}_{i}, \tilde{\tau}_{i}, \tilde{\pi}_{i j}\right\rangle_{i, j \in I^{*}}$ Let st : ${ }^{*} X \rightarrow X$ be the standard part map in the limit topologr, and for each $i \in I$ let st $i$ be the standard part map st $:_{i} \tilde{X}_{i} \rightarrow X_{i}$. It is easy to see that if st $(x)$ is defined, then st $\left(\tilde{\pi}_{i}(x)\right)$ is defined for all $i \in I$, and $\operatorname{st}(x)=\left\{s t_{i}\left(\tilde{\pi}_{i}(x)\right)\right\}_{i \in I^{\circ}}$ On the other hand, if $\operatorname{st}_{i}\left(\tilde{\pi}_{i}(x)\right)$ is defined for all $i \in I$, then $\operatorname{st}_{i}\left(\tilde{\pi}_{i}(x)\right)=\pi_{i j}\left(\operatorname{st}_{j}\left(\tilde{\pi}_{j}(x)\right)\right)$ for $j \geq i$, and $s t(x)$ is defined and equal to $\left\{\operatorname{st}_{i}\left(\tilde{\pi}_{i}(x)\right)\right\}_{i \in I}$.

Let us recall a few simple facts of nonstandard topology: If $\mathrm{X}$ is a Hausdorff space and st : ${ }^{*} \mathrm{X} \rightarrow \mathrm{X}$ the standard part map, then ${ }^{*} \mathrm{G} \supset \mathrm{st}^{-1}(\mathrm{G})$ for all open sets $\mathrm{G}$, and ${ }^{*} \mathrm{~K} \subset \mathrm{st}{ }^{-1}(\mathrm{~K})$ for all compact sets $K_{0}$ We are working with polysaturated models; and if $A$ is an internal subset of ${ }^{*} X$ in such a model, ther st(A) is closed (see e.g. Stroyan and Iuxemburg [18], page 201)。

Having now coinpleted our survey of the topological prerequisites, we turn to measure theory: By a Radon measure $\mu$ on a topological space $\langle\Psi, \sigma\rangle$, we shall mean a Borel probability measure on $Y$ such that for all Borel sets $B$ and all $\epsilon>0$, there is a compact set $K \subset B$ and an open set $G \supset B$ such that

$$
\mu(G)-\varepsilon<\mu(B)<\mu(K)+\epsilon_{0}
$$


By a cylindrical measure $\left\{\mu_{i}\right\}_{i \in I}$ on a projective system $\left\langle x_{i}, \tau_{i}, \pi_{i j}\right\rangle_{i, j \in I}$ of Hausdorff-spaces, we mean a net $\left\{\mu_{i}\right\}_{i \in I}$ of Radon measures on the $x_{i}$ such that $\pi_{i j}\left(\mu_{j}\right)=\mu_{i}$. We write $\left\{\tilde{\mu}_{i}\right\}_{i \in{ }^{*} I}$ for the ${ }^{*}$-version ${ }^{*}\left(\left\{\mu_{i}\right\}_{i \in I}\right)$. A cylinder set in $X$ is a set of the form $\pi_{i}^{-1}(B)$ where $B$ is a Borel set in $X_{i}$, $i \in I$ 。

The problem we consider in this section is the following: A cylindrical measure obviously induces a finitely additive measure on the cylinder sets, and using the Kolmogorov Extension Theorem this may be extended to a $\sigma$-additive measure. But can it be extended to a Radon measure on $X$ ? This is far from obvious since the $\sigma$-algebra generated by the cylinder sets is much smaller in general than the Borel algebra on $X_{0}$ However, Prohorov's theorem gives a simple characterization of when such an extension exists.

Before we turn to the proof of the theorem, we must know how Radon measures arise from Loeb-measures; the study of such problems was initiated by Anderson [2], and has since been studied by Henson [3] and Loeb [12]. We shall only need the simple

Iemma 1: Let $\langle\mathrm{X},(\mathbb{B}, \mu\rangle$ be a Borel probability space on a Hausdorff space. Assume that for each $\varepsilon>0$ there is a compact $K_{\epsilon}$ with $\mu\left(K_{\epsilon}\right)>A_{-} \varepsilon_{0}$ Let $\langle Z, I(G), L(P)\rangle$ be the Loeb-space of a nonstandard probability space $\langle Z, G, P\rangle$, and let $\theta: Z \rightarrow X$ be a partial mapping such that $\theta^{-1}(B) \in L(G)$ and $L(P)\left(\theta^{-1}(B)\right)=$ $\mu(B)$ for all $B \in B$. Assume further that $\theta(A)$ is closed for all $A \in G$. Then $\mu$ is a Radon measure.

Proof: Let $B \in B$ be given; then $\theta^{-1}(B) \in L(G)$ and for each $\epsilon>0$ there is an $A \in G, A \subset \theta^{-1}(B)$ such that 
$\mathrm{I}(\mathrm{P})\left(\theta^{-1}(\mathrm{~B})\right)-\mathrm{I}(\mathrm{P})(\mathrm{A})<\frac{\epsilon}{2}$. But $\theta(A) \subset \theta \theta^{-1}(B)=B$ is closed, and $\mu(\theta(A))=I(P)\left(\theta^{-1}(\theta(A))\right) \geq I(P)(A)$. Hence $\theta(A) \cap K_{\epsilon / 2}$ is a compact set conteined in B with

$$
\mu(B)-\mu\left(\theta(A) \cap K_{\epsilon / 2}\right)<\varepsilon
$$

The outer regularity follows by applying this result to $\mathrm{X}-\mathrm{B}$, and hence the lemrna is proved.

We may now prove Prohorov's theorem:

Theorem 2 (Prohorov): Let $\left\{\mu_{i}\right\}_{i \in I}$ be a cylindrical measure on a projective system $\left\langle\mathrm{x}_{i}, \tau_{i}, \pi_{i j}\right\rangle$ of Hausdorff spaces. The following is a necessary and sufficient condition that there exists a Radon measure $\mu$ on $X$ such that $\pi_{i}(\mu)=\mu_{i}$ for all $i \in I$ :

$\left(^{*}\right)$ For all $\epsilon>0$, there is a compact $K_{\epsilon} \subset X$ such that $\mu_{i}\left(\pi_{i}\left(K_{\epsilon}\right)\right) \geq 1-\epsilon$ for all $i \in I$.

Proof: That (*) is necessary is almost obvious: Let $\epsilon>0$ be given; since $\mu$ is Radon there exists a compact $K \subset X$ such that $\mu(K) \geq 1-\varepsilon_{0}$ Since $K$ is compact, so is $\pi_{i}(K)$, and hence $\pi_{i}(K)$ is $\mu_{i}$-measurable. But then

$$
\mu_{i}\left(\pi_{i}(K)\right)=\mu\left(\pi_{i}^{-1} \pi(K)\right) \geq \mu(K)=1-\epsilon
$$

which proves the necessity of $\left({ }^{*}\right)$.

We now turn to the sufficiency, and divide the proof into four steps.

1. Construction of $\mu$ : Let $\omega \in{ }^{*} I$ be larger than all the elements in I - such an element exists by polysaturation - and define $\theta: \tilde{X}_{w} \rightarrow X$ by $\pi_{i}(\theta(x))=\operatorname{st}_{i}\left(\tilde{\pi}_{i \omega}(x)\right)$. Obviously 
$\theta \circ \tilde{\pi}_{\omega}=$ st, and hence $\theta$ maps internal sets on closed sets. Let $\left\langle\tilde{\mathrm{X}}_{\omega}, \mathrm{I}\left(\tilde{\tilde{B}}_{\omega}\right), \mathrm{I}\left(\tilde{\mu}_{\omega}\right)\right\rangle$ be the Loeb-space of $\left\langle\tilde{\mathrm{X}}_{\omega}, \tilde{B}_{\omega}, \tilde{\mu}_{\omega}\right\rangle$, where $\tilde{B}_{\omega}$ is the ${ }^{*}$-Borel-algebra of $\tilde{\mathrm{x}}_{\omega}$. Define

$$
\mu=\theta\left(I\left(\tilde{\mu}_{\omega}\right)\right)
$$

2. $\mu$ is a Borel-measure: We first show that if $K \subset X$ is compact, then $\theta^{-1}(K) \in I\left(\widetilde{C}_{\omega}\right)$ : The family $\left\{\pi_{i}^{-1} \pi_{i}(K)\right\}$ is decreasing, and so is $\mu_{i}\left(\pi_{i}(K)\right)$ since $\mu_{j}\left(\pi_{j}(K)\right) \leq \mu_{j}\left(\pi_{i j}^{-1} \pi_{i}(K)\right)=$ $\mu_{i}\left(\pi_{i}(K)\right)$. Let

then

$$
\beta=\lim _{i \in I} \mu_{i}\left(\pi_{i}(K)\right)
$$

$$
L\left(\tilde{\mu}_{\omega}\right)\left(\tilde{\pi}_{\omega}(* K)\right)=\beta,
$$

since $\omega$ is infinitely large。

Since $K$ is compact,

$$
\theta^{-1}(K)=\tilde{\pi}_{\omega} s t^{-1}(K) \supseteq \tilde{\pi}_{\omega}(* K),
$$

and thus $\theta^{-1}(K)$ contains a set of measure $\beta$.

Let $\varepsilon>0$ be given, and choose $i \in I$ such that $\mu_{i}\left(\pi_{i}(K)\right)<\beta+\frac{\epsilon}{2}$. Since $\mu_{i}$ is Radon there is a $G_{i} \in \tau_{i}$ such that $\pi_{i}(K) \subset G_{i}$ and $\mu_{i}\left(G_{i}\right)<\beta+\varepsilon_{0}$. Hence

$$
L\left(\tilde{\mu}_{\omega}\right)\left(\tilde{\pi}_{i \omega}^{-1}\left({ }^{*} G_{i}\right)\right)={ }^{0} \tilde{\mu}_{\omega}\left(\tilde{\pi}_{i \omega}^{-1}\left({ }^{*} G_{i}\right)\right)={ }^{0} \tilde{\mu}_{i}\left({ }^{*} G_{i}\right)<\beta+\varepsilon .
$$

Since $G_{i}$ is open, we also have

$$
\begin{aligned}
& \theta^{-1}(K)=\tilde{\pi}_{\omega} s t^{-1}(K) \subseteq \tilde{\pi}_{\omega} s t^{-1}\left(\pi_{i}^{-1} \pi_{i}(K)\right) \subseteq \tilde{\pi}_{\omega} s t^{-1}\left(\pi_{i}^{-1}\left(G_{i}\right)\right) \subseteq \tilde{\pi}_{\omega}^{*}\left(\pi_{i}^{-1} G_{i}\right)= \\
& \tilde{\pi}_{\omega} \tilde{\pi}_{i}^{-1}{ }^{*} G_{i}=\tilde{\pi}_{i \omega}^{-1}\left({ }^{*} G_{i}\right) .
\end{aligned}
$$

This proves that for all $\epsilon>0, \theta^{-1}(\mathrm{~K})$ is contained in a 
set of measure less than $\beta+\varepsilon$. Since $\left\langle\tilde{X}_{\omega}, I\left(\tilde{\beta}_{\omega}\right), L\left(\tilde{\mu}_{\omega}\right)\right\rangle$ is complete, it follows that $\theta^{-1}(K) \in I\left(\widetilde{B}_{\omega}\right)$, and that $I\left(\tilde{\mu}_{\omega}\right)\left(\theta^{-1}(K)\right)=$ $\lim _{i \in I} \mu_{i}\left(\pi_{i} K\right)$ 。

If now $F \subset X$ is closed, $F \cap K_{1 / n}$ is compact, and hence $\theta^{-1}\left(U\left(F \cap K_{1 / n}\right)\right) \in L\left(\tilde{B}_{\omega}\right)$. The set $\theta^{-1}\left(F-\underset{n \in \mathbb{N}}{U}\left(F \cap K_{1 / n}\right)\right)$ is a subset of the null-set $\tilde{X}_{w}-\theta^{-1}\left(U K_{1 / n}\right)$, and is consequently in $L\left(\widetilde{B}_{\omega}\right)$. Hence $\theta^{-1}(F) \in I\left(\widetilde{B}_{\omega}\right)$ for all closed $F$, and it follows that $\mu$ is a Borel-measure.

2. $\mu$ is a Radon measure: Since $\theta$ maps internal sets on closed sets, this follows from Lemma 1 , and $\underline{2}$ above.

4. Consistency conditions: It remains to show that if $A \in B_{i}$, then $\mu\left(\pi_{i}^{-1}(A)\right)=\mu_{i}(A)$ : Let $\epsilon>0$ be given, and choose a neighbourhood $G$ of $A$ in $\tau_{i}$ such that $\mu_{i}(G) \leq \mu_{i}(A)+\varepsilon_{0}$ Since $\pi_{i}^{-1}(G)$ is open: $\mu\left(\pi_{i}^{-1}(A)\right)=L\left(\tilde{\mu}_{\omega}\right)\left(\theta^{-1}\left(\pi_{i}^{-1}(A)\right)=\right.$ $L\left(\tilde{\mu}_{\omega}\right)\left(\tilde{\pi}_{\omega} s t^{-1}\left(\pi_{i}^{-1}(A)\right)\right) \subseteq L\left(\tilde{\mu}_{\omega}\right)\left(\tilde{\pi}_{\omega} s t^{-1}\left(\pi_{i}^{-1}(G)\right)\right) \subseteq L\left(\tilde{\mu}_{\omega}\right)\left(\tilde{\pi}_{\omega} \tilde{\pi}_{i}^{-1}\left({ }^{*} G\right)\right)=$ $I\left(\tilde{\mu}_{\omega}\right)\left(\tilde{\pi}_{i \omega}^{-1}\left({ }^{*} G\right)\right)=\mu_{i}(G) \leq \mu_{i}(A)+\varepsilon_{0}$

This implies that $\mu\left(\pi_{i}^{-1}(A)\right) \leq \mu_{i}(A)$, but since the same must apply to the complement of $A$, we have $\mu\left(\pi_{i}^{-1}(A)\right)=\mu_{i}(A)$. This proves the theorem.

The above proof gives a straightforward construction of the limit measure as the "standard part" of the Loeb-measure of an infinitely large element in $\left\{\tilde{\mu}_{i}\right\}_{i \in I^{*}}$. For aplications of Prohorov's theorem, the reader should consult Schwartz [17]; we shall only give one which will be useful in the next section. We shall need the following version of the thoorem: 
Corollary 3: Let $\left\{\mu_{i}\right\}_{i \in I}$ be a cylindrical measure on $\left\langle X_{i}, \tau_{i}, \pi_{i j}\right\rangle$. Let $Y$ be a set, and assume that for each $i \in I$, $P_{i}$ is a mapping of $Y$ on $X_{i}$ such that $P_{i}=\pi_{i j}{ }^{\circ} P_{j}$, when $i<j$. Let $\sigma$ be the weakest topology on $Y$ making all the $P_{i}$ continuous. Then there is a Radon measure $\nu$ on $Y$ such that $\nu\left(P_{i}^{-i}(A)\right)=\mu_{i}(A)$ for all $i \in I$ and $A \in B_{i}$ if and only if: (**) For a.l $\epsilon>0$ there is a compact set $K_{\epsilon} \subset Y$ such that $\mu_{i}\left(P_{i}\left(K_{\epsilon}\right)\right)>1-\varepsilon$ for all $i \in I_{0}$

Proof: The necessity of $\left(*^{*}\right)$ is proved as in the proof of the theorem.

To prove the sufficiency, define $\mathrm{S}: \mathrm{Y} \rightarrow \mathrm{X}$ by $\mathrm{S}(\mathrm{y})=$ $\left\{\mathrm{P}_{i}(\mathrm{y})\right\}_{i \in I} \quad \mathrm{~S}$ is continuous, and by construction $\mathrm{P}_{i}=\pi_{i}{ }^{\circ} \mathrm{S}$. Thus $S\left(K_{\epsilon}\right)$ is compact and $\mu_{i}\left(\pi_{i}\left(S\left(K_{\epsilon}\right)\right)\right)=\mu_{i}\left(P_{i}\left(K_{\epsilon}\right)\right)>1-\epsilon$ for all $i \in I$. Using the theorem, we have a Radon measure $\mu$ on $X$ such that $\mu_{i}\left(\pi_{i}^{-1}(A)\right)=\mu_{i}(A)$. Defining $\nu\left(S^{-1}(A)\right)=\mu(A)$, we prove the corollary.

We turn to our application: Let $\mathrm{H}$ be a real separable Hilbert-space, and let $I$ be the set of all finite dimensional subspaces ordered by inclusion. If $E, F \in I, E=F$, let $P_{E}: H \rightarrow E$ and $P_{E, F}: F \rightarrow E$ be the projections. $\left\langle E, \tau_{E}, P_{E, F}\right\rangle$ is obviously a projective system of Hausdorff spaces, when $\tau_{E}$ is the norm topology。

Corollary 3 should now tell us when a cylindrical measure $\left\{\mu_{E}\right\}_{E \in I}$ on $\left\langle E, \tau_{e}, P_{E, F}\right\rangle$ can be extended to a measure on $H_{\text {. }}$ We introduce the following notation: 


$$
E_{I}=\{x \in H:\|x\| \leq r\}
$$

is the closed ball of radius $r$ around the origin. It is easy to check that these balls are in the $\sigma$-algebra generated by the cylinder-sets. Define

$$
\alpha_{r}^{E}=\mu_{E}\left(P_{E}(B)\right) \text { for } E \in I \text {. }
$$

For fixed $r$, the set $\left\{\alpha_{r}^{E}\right\}_{E \in I}$ is decreasing, and we let

$$
a_{r}=\inf _{E} \alpha_{r}^{E} \text {. }
$$

The function $r \rightarrow \alpha_{r}$ is increasing, and we may define

$$
\alpha=\lim _{r \rightarrow \infty} \alpha{ }^{\circ}
$$

Corollary 4: A cylindrical measure $\left\{\mu_{E}\right\}$ on $H$ has a $\sigma$-additive extension $\nu$ if and only if $\alpha=1$. The extension is a Radon measure.

Proof: Assume the extension $\nu$ exists. Then $B_{r} \subset P_{E}^{-1} P_{E}\left(B_{r}\right)$ for all $E \in I$, and hence $\nu\left(B_{r}\right) \leq \alpha_{r}$. Since $\nu(H)=\nu\left(\underset{n \in \mathbb{N}}{\cup} B_{n}\right)=1$, $a_{r} \rightarrow 1$, and hence $\alpha=1$.

Assume now that $\alpha=1$. The weakest topology on $\mathrm{H}$ making all the finite dimensional projections continuous, is the weak topology. But on $H$ the weak topology and the weak-* topology coincide, and hence by the Banach-Alaoglu Theorem (see e.g. Reed and Simon [15], page 115) all the balls $B_{r}$ are compact in this topology. Given $\varepsilon>0$, choose $r_{\epsilon}$ such that $\alpha_{r_{\epsilon}}>1-\varepsilon$. Then $\mu_{E}\left(P_{E}\left(B_{r_{\varepsilon}}\right)\right)>1-\varepsilon$ for all $E \in I$. Applying Corollary 3 with $\mathrm{K}_{\epsilon}=\mathrm{B}_{\mathrm{r}_{\varepsilon}}$, we see that an extension $\nu$ exists, and is a Radon measure with respect to the weak topology. But since the balls 
are $\nu$-measurable, $\nu$ is also a Radon measure with respect to the norm topology.

From the nonstandard point of view, Corollary 4 has the following interpretation: Let $\left\{e_{n}\right\}$ be an orthonormal basis for $H$, and let $\left\{\tilde{e}_{n}\right\}_{n} \in^{*} \mathbb{H}$ be its *-version. Let $E$ be the hyperfinite dimensional subspace of ${ }^{*} \mathrm{H}$ generated by $\tilde{\mathrm{e}}_{1}, \ldots, \widetilde{\mathrm{e}}_{\eta}$ for some $\eta \in{ }^{*} \mathbb{N} \backslash \mathbb{N}$. It is then easy to see that $\alpha_{S} \leq{ }^{0} \alpha_{r} \leq \alpha_{r}$ for $s \in \mathbb{R}$, $s<r$, and thus ${ }^{\circ} \alpha \underset{r}{E} \rightarrow \alpha$ as $r \rightarrow \infty$. Hence $\mu$ has a $\sigma$-additive extension if and only if $L\left(\tilde{\mu}_{E}\right)$ has support on the elements of finite norm in $E$; i.e. the near-standard elements in the weak topology. We may perhaps say that a limit measure $I\left(\tilde{\mu}_{\mathrm{E}}\right)$ always exists; the problem is whether it has near-standard support so that we can push it down to $H_{0}$. In the following two sections we shall try to elucidate this theme.

\section{Characteristic functionals on Hilbert apaces:} Sazonov's theorem.

A famous theorem by $S$. Bochner characterizes the class of Fourier-transforms of probability measures on $\mathbb{R}^{n}$ as those functions $\varphi: \mathbb{R}^{\mathrm{n}} \rightarrow \mathrm{C}$ that take the value one in the origin, are positive definite and continuous. It is natural to guess that this theorem has a generalization to Hilbert spaces, and since most Hilbert space topologies coincide on finite dimensional spaces, one might conjecture that the task is "only" to find the right notion of continuity. That norm-continuity is not the right notion is easy to see; but in $1958 \mathrm{~V} . \mathrm{V}$. Sazonov [16] proved that the right topology is the one generated by the 
Hilbert-Schmidt operators.

We shall now give a nonstandard proof of this theorem:

Let $H$ be a real separable Hilbert space; a Hilbert-Schmidt operator $A: H \rightarrow H$ is a linear operator such that $\sum_{n=1}^{\infty}\left\|A_{n}\right\|^{2}<\infty$ for any orthonormal basis $\left\{e_{n}\right\}$ in $H_{0}$. It is easy to see that the sum $\Sigma\left\|A e_{n}\right\|^{2}$ is independent of which orthonormal basis we choose.

The sets of the form $\left\{x \in H:\left\|A_{1} x\right\|<\delta_{1}, \ldots,\left\|A_{m} x\right\|<\delta_{m}\right\}$ where $A_{1}, \ldots, A_{m}$ are Hilbert-Schmidt operators, and $\delta_{1}, \ldots, \delta_{m}$ are positive real numbers, form a basis for the Hilbert-Schmidt topology. By polar decomposition, any Hilbert-Schmidt operator A can be written as the product UT of an partial isometry $U$, and a symmetric, positive Hilbert-Schmidt operator T. Hence we may assume that $A_{1}, \ldots, A_{m}$ above are symmetric. Moreover, if $A_{1}, A_{2}$ are Hilbert-Schmidt operators, then there exists a Hilbert-Schmidt operator $B=\sqrt{A_{1}^{*} A_{1}+A_{2}^{*} A_{2}}$ such that $\|B x\|<\delta$ implies $\left\|A_{1} x\right\|<\delta$ and $\left\|A_{2} x\right\|<\delta$. Thus we may replace the sequence $A_{1}, \ldots, A_{m}$ above with a single, symmetric $H_{0}-S_{0}$ operator $A$ and still have a basis for the topology.

Let $\varphi: H \rightarrow C$ with $\varphi(0)=1$. Then $\varphi$ is continuous in 0 if and only if for all $\epsilon \in \mathbb{R}, \varepsilon>0$, there exists a symmetric Hilbert-Schmidt operator $A$ and a $\delta \in \mathbb{R}, \delta>0$, such that if $\|A x\|<\delta$ then $|\varphi(x)-1|<\varepsilon$. Taking the *-transfer of this, we get

$$
\forall \mathrm{x} \in{ }^{*} \mathrm{H}\left(\left\|^{*} \mathrm{Ax}\right\|<\left.\delta \rightarrow\right|^{*} \varphi(\mathrm{x})-1 \mid<\varepsilon\right) \text { 。 }
$$

By the internal definition principle this is equivalent to that for all $\varepsilon \in \mathbb{R}, \varepsilon>0$, there is a symmetric $H_{0}-S$. operator A such that

$$
\forall x \in^{*} \mathrm{H}\left(\left.\left\|^{*} \mathrm{AXX}\right\| \approx 0 \rightarrow\right|^{*} \varphi(\mathrm{x})-1 \mid<\epsilon\right)
$$


To prove that $\varphi$ is discontinuous in 0 , it is thus enough to find a positive $\in \in \mathbb{R}$, such that for all symmetric $H_{0}-S_{\bullet}-$ operators $A$ there is an $x \in{ }^{*} H$ with $\left\|^{*} A x\right\| \approx 0$ and $\left|{ }^{*} \varphi(x)-1\right| \geq \epsilon$ 。

To prove the theorem, we shall need the following notions: Let $\eta \in^{*} \mathbb{N} \backslash \mathbb{N}$, and define $\Omega=\{-1,1\}^{\eta}$, and let $P$ be the uniform *-probability measure on $\Omega ; P\{\omega\}=\left(\frac{1}{2}\right)^{\eta}$. By a symmetric binary martingale $x: \Omega \rightarrow{ }^{*} \mathbb{R}$ we shall mean a ${ }^{*}$-stochastic variable $x$ defined by $x(\omega)=\sum_{i=1}^{\eta} w_{i} y_{i}$, where $\left\{y_{i}\right\}_{i \leq \eta}$ is an internal sequence of hyperreal numbers. The quadratic variation of $x$ is defined to be $[x]=\sum_{i=1}^{\eta} \mathrm{y}_{i}^{2}$ (This should look familiar to readers acquainted with Anderson's Brownian motion [1]).

Lemma 5: Let $x$ be a symmetric binary martingale, and put $\gamma^{2}=[x]$. If $\gamma$ is finite, $x$ is finite $a_{0} e_{0}$ in the Loebmeasure. If $\gamma$ is infinite there is a set of measure $\geq \frac{1}{2}$ where $|x| \geq \sqrt{\gamma}$.

Proof: Assume that $\gamma$ is finite. Using that the $w_{i}$ are independent with mean zero and variance one, we get

$$
\left.E\left(x^{2}\right)=E\left(\sum_{i=1}^{\eta} w_{i} y_{i}\right)^{2}\right)=\sum_{i, j=1}^{\eta} y_{i} y_{j}\left(w_{i} \omega_{j}\right)=\sum_{i=1}^{\eta} y_{i}^{2}=\gamma^{2} .
$$

Consequently $x$ is finite $L(P)-a_{0} e$.

Assume so that $\gamma$ is infinite. We first consider the case where there is a $j \in\{1,2, \ldots, \eta\}$ with $\left|y_{j}\right| \geq \sqrt{\gamma}$, say $y_{j} \geq \sqrt{\gamma}$. Then either $\sum_{i \neq j} w_{i} \mathrm{y}_{i} \geq 0$ or $\sum_{i \neq j}\left(-w_{i}\right) \mathrm{y}_{i} \geq 0$, so for each $w$ such that $\sum_{i=1}^{n} w_{i} y_{i}<\sqrt{\gamma}$ there is a $w^{\prime}=\left(-w_{1},-w_{2}, \ldots, w_{j}, \ldots,-w_{\eta}\right)$ such that $\sum_{i=1}^{n} w_{i}^{\prime} y_{i} \geq \sqrt{\gamma}$. Hence $x \geq \sqrt{\gamma}$ with probability $\geq \frac{1}{2}$. A similar argument applies for $\mathrm{y}_{j} \leq-\sqrt{\gamma}$. 
Assume so that $\gamma$ is infinite, but $\mid y_{j} k \sqrt{\gamma}$ for all $j \cdot$ Then $\left[\frac{X}{\gamma}\right]=1$, and by the first part of the proof $X / \gamma$ is finite a.e.. Moreover $y_{i} / \gamma \approx 0$ for all $i$. We calculate the characteristic function of the randcm variable ${ }^{\circ}(X / \gamma)$ :

$$
\begin{aligned}
& \int e^{i^{0}(X / Y) z} d L(P)=\int e^{i(x / Y) z} d P=\int e^{i} j_{j=1}^{\eta \omega_{j} \bar{y}_{j} z} \frac{\gamma}{\gamma} d P \\
& =\cdot \int \prod_{j=1}^{\eta} e^{i \frac{\omega_{j} y_{j} z}{\gamma}} d P=\left(\prod_{j=1}^{n} \int e^{i \frac{\omega_{j} y_{j} z}{\gamma}} d P\right)=\prod_{j=1}^{n} \frac{e^{i \frac{y_{j} z}{\gamma}}+e^{-i \frac{y_{j} z}{\gamma}}}{2}
\end{aligned}
$$

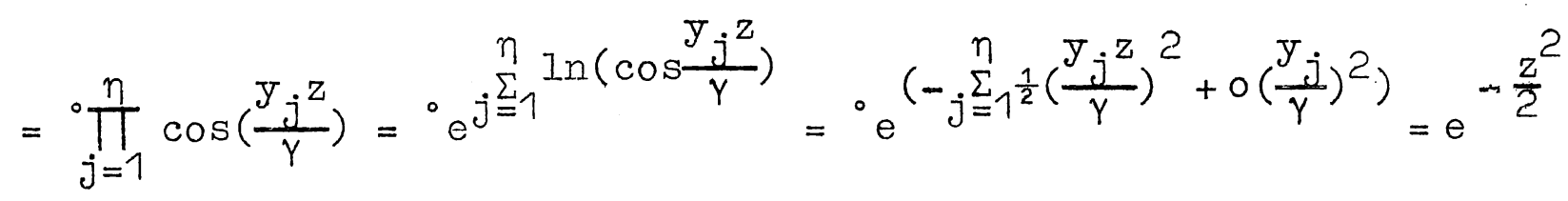

where we have used the independence of $w_{i}$ and $w_{j}$ for $i \neq j$, and the Taylor-expansion of $\ln (\cos x)$.

This tells us that ${ }^{\circ}(x / y)$ is gaussian distributed with variance 1. Hence the probability that $\left|\frac{X}{y}\right| \geq \frac{\lambda}{\sqrt{\bar{y}}}$ is larger than $\frac{1}{2}$, and so is the probability that $|x| \geq \sqrt{\gamma}$. This proves the lemma.

The proof of part (b) of the following proposition contains the heart of the argument:

Proposition 6: Let $\left\{\mu_{E}\right\}$ be a cylindrical measure on a real, separable Hilbert space H. Then (recall the $\alpha$ of Corollary 4):

(a) If $\alpha=1$, then for each positive $\in \in \mathbb{R}$ there is a symmetric Hilbert-Schmidt operator $T$ on $H$, and a $r_{e} \in \mathbb{R}$ such that $\nu\left(\mathrm{B}_{\mathrm{r}_{\epsilon}}\right)>1-\epsilon$, and for $\mathrm{x} \in{ }^{*} \mathrm{H}:\left\|^{*} \mathrm{Tx}\right\| \approx 0 \Rightarrow\langle\mathrm{x}, \mathrm{y}\rangle \approx 0$ for $L\left({ }^{*} \nu\right)$-almost all $y \in \in^{*} \mathrm{~B}_{\epsilon}{ }^{\circ}$ 
(b) If $a<1$, there is for each symmetric Hilbert-Schmidt operator $T$ on $H$ a set $B$ with ${ }^{*} \mu(B)>\frac{1-\alpha}{4}$, a $\gamma \in{ }^{*} \mathbb{N} \backslash \mathbb{N}$, and an $\mathrm{x} \in{ }^{*} \mathrm{H}$ such that

$$
\left\|^{*} \mathrm{Tx}\right\| \approx 0 \text { and }|\langle\mathrm{x}, \mathrm{y}\rangle|>\gamma^{\frac{1}{2}} \text { for } \mathrm{y} \in \mathrm{B}
$$

Proof: (a) This is the standard argument, adapted from Kuo [10]: Let $r_{\epsilon}$ be so large that $\nu\left(B_{r_{\epsilon}}\right)>1-\epsilon$, and consider the bilinear form $\int_{\mathrm{B}_{\mathrm{r}_{\epsilon}}}\langle\mathrm{x}, \mathrm{z}\rangle\langle\mathrm{y}, \mathrm{z}\rangle \mathrm{d} \nu(\mathrm{z})$. It is.. continuous since

$$
\left|\int_{\mathrm{B}_{\epsilon}}\langle x, z\rangle\langle y, z\rangle d \nu(z)\right| \leq\|x\|\|y\| \int_{\mathrm{B}_{r_{\epsilon}}}|z|^{2} d \nu(z) \leq\|x\|\|y\|\left\|r_{\epsilon}\right\|^{2} .
$$

Consequently there is a symmetric, continuous, positive operator $S$ such that

Now

$$
\langle\mathrm{Sx}, \mathrm{y}\rangle=\int_{\mathrm{B}_{\epsilon}}\langle\mathrm{x}, \mathrm{z}\rangle\langle\mathrm{y}, \mathrm{z}\rangle \mathrm{d} \nu(\mathrm{z})
$$

$$
\sum_{n=1}^{\infty}\left\langle S e_{n}, e_{n}\right\rangle=\sum_{n=1}^{\infty} \int_{r_{\epsilon}}^{\infty}\left\langle x, e_{n}\right\rangle^{2} d \nu(x)=\int_{B_{r_{\epsilon}}} \sum_{n=1}^{\infty}\left\langle x, e_{n}\right\rangle^{2} d \nu(x)=\int_{B_{r_{\epsilon}}}\|x\|^{2} d \nu(x) \leq r_{\varepsilon}^{2},
$$

showing that $S$ is a trace-class operator. Hence $S=T \circ T$ for some symmetric Hilbert-Schmidt operator $T$, and we get

$$
\|\mathrm{TX}\|^{2}=\int_{\mathrm{B}_{\epsilon}}\langle\mathrm{x}, \mathrm{z}\rangle^{2} \mathrm{~d} \nu(z)
$$

\section{(a) now follows from the *-version of this.}

(b) Let $\left\{e_{n}\right\}_{n \in \mathbb{I}}$ be an orthonormal basis of eigenvectors for $T$, and let $\left\{\tilde{e}_{n}\right\}_{n \leq \eta}$ be a hyperfinite initial segment of ${ }^{*}\left(\left\{e_{n}\right\}_{n \in \mathbb{N}}\right)$. Assume ${ }^{*} \widetilde{T}_{\tilde{e}_{n}}=\beta_{n} \widetilde{e}_{n}$, then ${ }_{n=1}^{\infty} \beta_{n}^{2}<\infty$. Let $E_{T}$ be 
the hyperfinite dimensional subspace of ${ }^{*} H$ generated by $\{\tilde{e}\}_{n \leq \eta}$. Choose $\gamma \in{ }^{*} \mathbb{N} \backslash \mathbb{N}$ such that

$$
\tilde{\mu}_{E_{T}}\left\{y \in E_{T}:\|y\| \geq \gamma^{5 / 4}\right\} \geq \frac{1-\alpha}{2}
$$

such a $\gamma$ exists by definition of $\alpha$ and the intermal definition principle.

Our element $x$ shall be of the form $x=\sum_{n=1}^{n} \frac{\omega_{n}}{\gamma^{1 / 4}} \widetilde{e}_{n}$, where $\omega_{n}= \pm 1$ for all $n$. Obviously $\left\|^{*} T x\right\|^{2}=\sum_{n=1}^{n} \frac{\beta^{2}}{\gamma^{\frac{1}{2}}} \approx 0$. We now use Lemma 5 to choose the sequence $\left\{w_{n}\right\}$ such that $\langle x, y\rangle$ is infinite on a set of $\mathrm{y}^{\prime} \mathrm{s}$ of large enough measure:

If $y$ is such that $\|y\|_{E_{T}}^{2}=\sum_{n=1}^{n} y_{n}^{2} \geq \gamma^{5 / 2}$, then $\sum_{n=1}^{n}\left(\frac{w_{n} y_{n}}{\gamma^{1 / 4}}\right)^{2} \geq \gamma^{2}$. By Lemma 5 is then $\left|\Sigma \frac{{ }^{\omega}{ }_{n} y_{n}}{\gamma^{1 / 4}}\right| \geq \gamma^{\frac{1}{2}}$ for all $\omega$ in a set of measure $\geq \frac{1}{2}$. Let now

$$
A=\left\{(\omega, y): \sum_{n=1}^{\eta} \frac{w_{n} y_{n}}{\gamma^{1 / 4}} \geq \gamma^{\frac{1}{2}}\right\}
$$

and apply Fubini's Theorem to the characteristic function $K_{A}$ :

$$
\begin{gathered}
\int\left[\int \mathrm{K}_{\mathrm{A}}(\omega, y) d \tilde{\mu}_{\mathrm{E}_{\mathrm{T}}}(y)\right] \mathrm{dP}(\omega)=\int\left[\int \mathrm{K}_{\mathrm{A}}(\omega, \mathrm{y}) \mathrm{dP}(\omega)\right] \mathrm{d} \tilde{\mu}_{\mathrm{E}_{\mathrm{T}}}(y) \geq \\
\geq \frac{1}{2} \tilde{\mu}_{\mathrm{E}_{\mathrm{T}}}\left\{y \in \mathrm{E}_{\mathrm{T}}:\|y\| \geq \gamma^{5 / 4}\right\} \geq \frac{1-\alpha}{4} .
\end{gathered}
$$

Hence there is a $\tilde{\mu} \in \Omega$ such that $\tilde{\mu}_{E_{T}}\left\{y: \mid \sum_{n=1}^{\eta} \frac{\tilde{\omega}_{n} y_{n}}{\gamma^{1 / 4}} \geq \gamma^{\frac{1}{2}}\right\} \geq \frac{1-\alpha}{4}$. Choose $x=\sum_{n=1}^{n} \frac{\tilde{\omega}_{n}}{\gamma / 4} \tilde{e}_{n} \cdot$ Then

$$
{ }^{*} \mu\left\{y \in{ }^{*} H:|\langle x, y\rangle| \geq \gamma^{\frac{1}{2}}\right\}=\tilde{\mu}_{E_{T}}\left\{y \in E_{T}:\left|\sum_{n=1}^{\eta} \frac{\tilde{w}_{n}}{\gamma^{1 / 4}} y_{n}\right| \geq \gamma^{\frac{1}{2}}\right\} \geq \frac{1-\alpha}{4},
$$

which proves (b). 
It is now easy to prove

Theorem 7 (Sazonov): Let $\varphi: H \rightarrow C$ be a complex-valued function on a real, separable Hilbert space. Then $\varphi$ is the Fourier-transform of a Borel probability measure on $H$ if and only if: $\varphi(0)=1, \varphi$ is positive definite and continuous in the Hilbert-Schmidt topology.

Proof: Assume $\varphi(x)=\int e^{i\langle x, y\rangle} d \mu(y)$, where $\mu$ is a probability measure on $H ;$ then $\varphi(0)=1$ and $\varphi$ is positive definite. Let $\epsilon>0$ be given. Since $\alpha=1$, we may apply Proposition 6(a) to get a symmetric Hilbert-Schmidt operator $T$ and a set $\mathrm{B}_{r_{\epsilon / 3}}$ with $\mu\left(B_{r_{\epsilon / 3}}\right)>1-\varepsilon / 3$ such that $\left\|^{*} \mathrm{Tx}\right\| \approx 0 \Rightarrow\langle\mathrm{x}, \mathrm{y}\rangle \approx 0$ for almost all $\mathrm{y} \in{ }^{*} \mathrm{~B}_{\mathrm{r}_{\epsilon / 3}}$. Consequently $\mathrm{e}^{i(\mathrm{x}, \mathrm{y})} \approx 1$ on a set of measure $\geq 1-\epsilon / 3$, and hence ||$^{*} \varphi(\mathrm{x})-1 \mid<\epsilon$. By our nonstandard characterization of $H_{0}-S_{0}$ continuity, it follows that $\varphi$ is continuous in 0 . Since $\varphi$ is positive definite this implies that $\varphi$ is $\mathrm{H}_{0}-\mathrm{S}_{0}$-continuous everywhere.

Assume now that $\varphi$ is a positive definite, $H_{0}-$ S.-continuous function with $\varphi(0)=1$. Using Bochner's Theorem on the finite djmensional subspaces of $\mathrm{H}$, we see that $\varphi$ is the Fourier-transform of a cylindrical measure $\left\{\mu_{\mathrm{E}}\right\}$ on $\mathrm{H}$. We shall show that $\alpha=1$ by proving that if $a<1$, then $\varphi$ can not be $H_{0}-S_{0}-$ continuous o

So assume $\alpha<1$ : Given a symmetric $H_{0}-S_{0}$-operator T, there exist by Proposition $6(\mathrm{~b})$ a set $B$ with ${ }^{*} \mu(B)>\frac{1-\alpha}{4}$, a $\gamma \in{ }^{*} \mathbb{I} \backslash \mathbb{N}$, and an $x \in{ }^{*} H$ such that

$$
\left\|^{*} \mathrm{Tx}\right\| \approx 0 \text { and }|\langle\mathrm{x}, \mathrm{y}\rangle| \geq \gamma^{\frac{1}{2}} \text { for } \mathrm{y} \in \mathrm{B} \text {. }
$$


Let $\lambda$ be the Lebesgue measure on $\mathbb{R}$. It is easy to see that for infinite $\mathrm{z}$

$$
{ }^{*} \lambda\left\{a \in^{*}[1,2]: a z \in \underset{k \in Z^{*}}{U}\left\{{ }^{*}\left[2 k \pi+\frac{\pi}{2}, 2 k \pi+\frac{3 \pi}{2}\right]\right\}\right\} \approx \frac{1}{2}>\frac{1}{3} \text {. }
$$

Define

$$
A=\left\{(a, y): a\langle x, y\rangle \in \underset{k \in * Z}{U}\left\{{ }^{*}\left[2 k \pi+\frac{\pi}{2}, 2 k \pi+\frac{3 \pi}{2}\right]\right\}\right\} .
$$

By Fubini's Theorem

$$
\int_{1}^{2}\left[\int_{B} K_{A}(a, y) d^{*} \mu(y)\right] d^{*} \lambda(a)=\int_{B}\left[\iint_{1}^{2} K_{A}(a, y) d^{*} \lambda(a)\right] d^{*} \mu(y) \geq \frac{1}{3} *^{*} \mu(B) \geq \frac{1-a}{12} \text {. }
$$

Hence there exist an a such that

$$
*_{\mu}^{*}\left\{y: a\langle x, y\rangle \in U\left\{{ }^{*}\left[<k \pi+\frac{\pi}{2}, 2 k \pi+\frac{3 \pi}{2}\right]\right\}\right\} \geq \frac{1-\alpha}{12} 。
$$

For such an a, $\operatorname{Re}\left(e^{i\langle a x, y\rangle}\right)=\cos (\langle a x, y\rangle) \leq 0$ for all $y$ in a set of measure $>\frac{1-\alpha}{12}$, and consequently

$$
\left|\int e^{i\langle a x, y\rangle} d^{*} \mu(y)-1\right| \geq \frac{1-\alpha}{12}
$$

Since $\left\|^{*} T(a x)\right\| \approx 0$, this implies that $\varphi$ is not $H_{0}-S_{0}$-continuous in 0 , and the theorein is proved.

What I would like to point out about the proof above, is the following: In this case the hard thing is to find out what happens when the limit measure does not exist. But in the nonstandard universe we do have a kind of limit measure $L\left(\tilde{\mu}_{F_{1}}\right)$, which lives on a space $E_{T}$ with all the algebraic and topological structure of a linear space. We can thus perform all the necessary calculations of Proposition 6 and Theorem 7 on this space. The argument could probably be carried through in a standard way working on the finite dimensional subspace of $H$, by picking suitable se- 
quences of elements and the right notion of convergence of measures, but the clarity of the argument would then most likely be lost in a haze of convergence results.

IV. Cylindrical measures extended to Banach spaces: Gross' theorem.

In the first section we inquired when a cylindrical measure $\left\{\mu_{E}\right\}$ on a Hilbert space can be extended to a countably additive measure $\mu$ on $\mathrm{H}$; and we saw - indirectly - how such a measure could be obtained from $\mathrm{I}\left(\tilde{\mu}_{E}\right)$ on a hyperfinite dimensional subspace $E$ of ${ }^{*} H$ using a standard part map. We may ask ourselves in cases where such an extension is not possible, whether we may find some other reasonable limit measure, perhaps living on a larger space. One way of doing this in a nonstandard setting would be to use a standard part map connected with a weaker topology; this would make more points near-standard. We shall now apply this strategy to prove a theorem of Gross [5] on the extension of cylindrical measures to Banach spaces.

Let $H$ be a real, separable Hilbert space with an inner product $\langle\cdot, \cdot\rangle$ generating a norm $\|\cdot\|$, and let $|\cdot|$ be another norm on $H_{0}$ An element $y \in{ }^{*} H$ is called $|\cdot|$-near-standard if for all $\epsilon \in \mathbb{R}, \epsilon>0$, there is an $x \in H$ with $|x-y|<\epsilon$. If $E$ is a hyperfinite dimensional subspace of ${ }^{*} \mathrm{H}$, define an equivalence relation $\sim_{E}$ on $E$ by $x \sim_{E} \mathrm{y} \Leftrightarrow|x-y| \approx 0$. Let $N_{1}$ (E) denote the $|\cdot|$-near-standard elements of $E$, and let ${ }^{\circ} \mathrm{E}=\left.\mathrm{Ns}\right|_{\mid}(\mathrm{E}) / \sim_{E}$ Let ${ }^{\circ}|\cdot|$ be the norm defined on ${ }^{\circ} \mathrm{E}$ by $|x|=$ st $|\tilde{x}|$, when $\tilde{x}$ is the equivalence class of $x_{0}$ 
Lemma 8: Let $E \epsilon^{*} I$ be such that $H \subset E$. Then $\left({ }^{\circ} E,{ }^{\circ}|\cdot|\right)$ is the completion of $\mathrm{H}$ in $|\cdot|$-norm.

Proof: If $\mathrm{x} \in \mathrm{H}$, identify it with its equivalence class $\tilde{\mathrm{x}}$ under $\sim_{E^{\circ}}$ By definition of $|\cdot|$-near-standard, $H$ is dense in ${ }^{\circ} E$, and it only remains to prove that $\%$ Let $\left\{\tilde{x}_{n}\right\}_{n \in \mathbb{N}}$ be a Cauchy-sequence in ${ }^{\circ} E$, and let for each $n \in \mathbb{N}$ $x_{n} \in \tilde{x}_{n}$. Then $\left\{x_{n}\right\}_{n \in \mathbb{N}}$ can be extended to an internal sequence $\left\{x_{n}\right\}_{n \in *} \mathbb{N}$ of elements from $E$ such that $\left|x_{n}-x_{m}\right| \approx 0$ for all $n, m \in{ }^{*} \mathbb{N} \backslash \mathbb{N}$. Let $\gamma \in{ }^{*} \mathbb{N} \backslash \mathbb{N}$, and let $\tilde{x}_{\gamma}$ be the equivalence class of $x_{\gamma}$. Since all $x_{n}$ are $|\cdot|$-near-standard, so is $x_{\gamma}$, and the sequence $\left\{\tilde{x}_{n}\right\}$ converges to $\tilde{x}_{\gamma^{*}}$ The lemma is proved.

From now on we write $B$ for ${ }^{\circ} E$ and $|\cdot|$ for ${ }^{\circ}||$; and we have hence shown the existence of a standard part map st $_{1 !}: N s_{\| !}(E) \rightarrow B$.

Let $\mathcal{F}$ be the set of finite dimensional projections in $H$. If $\mu=\left\{\mu_{F}\right\}_{F \in I}$ is a cylindrical measure on $H$, the norm || is called $\mu$-measurable if for all $\epsilon \in \mathbb{R}_{+}$there is a $P_{0} \in \mathscr{F}$ such that

$$
\mu\{|\mathrm{Px}|>\epsilon\}<\epsilon \text { for all } \mathrm{P} \in \mathcal{T}, \mathrm{P} \perp \mathrm{P}_{0} \text {. }
$$

Gross' theorem says that if || is $\mu$-measurable, then $\mu$ has an extension to Borel-measure on B. The key observation is the following:

Lemma 9: If $|\cdot|$ is $\mu$-measurable, then $\mathrm{L}\left(\tilde{\mu}_{E}\right)\left(N s_{1},(E)\right)=1$ ( $E$ is as in Lemma 8.)

Proof: Let

$$
A_{m}=\left\{x \in E: \exists v \in H\left(|v-x|<\frac{1}{m}\right)\right\} \text {. }
$$


If $P \in \mathcal{F}$, let $\mathrm{F}_{\Psi}^{\perp}$ be the orthogonal projection to ${ }^{*} \mathrm{P}$ in $\mathrm{E}_{\mathrm{o}}$ For $\mathrm{n}>\mathrm{m}$, find a $\mathrm{P} \in \mathcal{F}$ such that

$$
\tilde{\mu}_{E}\left\{\left|P_{E}^{\perp}(x)\right|>\frac{1}{n}\right\}<\frac{1}{n},
$$

by $\mu$-measurability of $1 \cdot 1$. Then the set

$$
\left\{x \in E: P(x) \in N s_{11}(E)\right\} \cap\left\{x:\left|P_{E}^{1}(x)\right| \leq \frac{1}{n}\right\}
$$

is contained in $A_{m}$, and has Loeb-measure $\geq 1-\frac{1}{n} \cdot{ }^{*}$ Since this holds for all $n>m, I\left(\tilde{\mu}_{E}\right)\left(A_{m}\right)=1$. But $N s_{1}(E)=\bigcap_{m} A$, and hence $I\left(\tilde{\mu}_{E}\right)\left(N s_{1},(E)\right)=1$, which proves the lemma.

To prove the theorem, it only remains to push $L\left(\tilde{\mu}_{E}\right)$ down to $B$ using the standard part map. However, we must first agree on what it means for a measure on $B$ to extend a cylindrical measure on $\mathrm{H}$ : Let $\mathrm{B}^{*}$ be the dual of $\mathrm{B}$. If $\mathrm{y}_{1}, \ldots, \mathrm{y}_{\mathrm{n}} \in \mathrm{B}^{*}$ and $A$ is a Borel-set in $\mathbb{R}^{n}$, then the set $\left\{x \in B:\left(y_{1}(x), \ldots, y_{n}(x)\right) \in A\right\}$ is called a cylinder set in $B$. We define a finitely additive measure $\hat{\mu}$ on the cylinder sets by

$$
\hat{\mu}\left\{x \in B:\left(y_{1}(x), \ldots, y_{n}(x)\right) \in A\right\}=\mu\left\{x \in H:\left(\left\langle x, y_{1}\right\rangle, \ldots,\left\langle x, y_{n}\right\rangle\right) \in A\right\}
$$

where we have identified $\mathrm{H}$ and $\mathrm{H}^{*}$ and embedded $\mathrm{B}^{*}$ in $\mathrm{H}^{*}$.

Theorem 10 (Gross): Let || be $\mu$-measurable. Then $\hat{\mu}$ has an extension to a $\sigma$-additive Borel-measure on $B$.

Proof: Let $E$ be as in Lemmas 8 and 9. Define a measure $\nu$ on $B$ by $\nu=s t_{\|}\left(L\left(\tilde{\mu}_{E}\right)\right)$; this is well-defined probability measure since $I\left(\tilde{\mu}_{E}\left(N s_{1},(E)\right)=1\right.$ by Lemma 9. To prove that $\nu$ is a Borel-measure, it is enough to prove that all balls

$$
B(a, r)=\{x \in B:|x-a|<r\}, a \in H,
$$

$\left.{ }^{*}\right) I\left(\tilde{\mu}_{E}\right)\left\{x \in E: P(x) \in \mathbb{N} s_{\|}(E)\right\}=0$ since $P \in \mathscr{F}$ (and not only ${ }^{*} \mathscr{F}$ ) 
are $\nu$-measurable, since $B$ is separable. But

$$
\operatorname{st}_{1}^{-1}(B(a, r))=\underset{n}{U}\left\{x \in E:|x-a|<r-\frac{1}{n}\right\} \cap N \mid
$$

which is $L\left(\tilde{\mu}_{E}\right)$-measurable, and hence $\nu$ is a Borel-measure. It remains to prove that $\nu$ and $\hat{\mu}$ agree on the cylinder sets: Since $\operatorname{st}_{11}^{-1}\left\{x \in B:\left(y_{1}(x), \ldots, y_{n}(x)\right) \in A\right\}=\left\{x \in E:\left(y_{1}(x), \ldots, y_{n}(x)\right) \in s t^{-1}(A)\right\}$, we have $\left.\nu\left\{x \in B:\left(y_{1}(x), \ldots, y_{n}(x)\right) \in A\right\}=L\left(\tilde{\mu}_{E}\right)\left\{x \in E:\left(y_{1}(x), \infty, y_{n}(x)\right) \in \operatorname{st}^{-1}(A)\right)\right\} 。$ But $E \subset{ }^{*} H$, and hence $y_{j}(x)=\left\langle x, y_{j}\right\rangle$ and so $\left.\left.\nu\left\{x \in B:\left(y_{1}(x), \ldots, y_{n}(x)\right) \in A\right)\right\}=L\left(\tilde{\mu}_{E}\right)\left\{x \in E:\left(\left\langle x, y_{1}\right\rangle, \infty,\left\langle x, y_{n}\right\rangle\right) \in s t^{-1}(A)\right)\right\}$. On the other hand $\hat{\mu}\left\{x \in B:\left(y_{1}(x), \ldots, y_{n}(x)\right) \in A\right\}=L\left(\tilde{\mu}_{E}\right)\left\{x \in E:\left(y_{1}(x), \ldots, y_{n}(x)\right) \in{ }^{*} A\right\}$ $=L\left(\tilde{\mu}_{E}\right)\left\{x \in E:\left(\left\langle x, J_{1}\right\rangle, \ldots,\left\langle x, y_{n}\right\rangle\right) \in{ }^{*} A\right\}$.

Keoping $\mathrm{y}_{1}, \ldots, \mathrm{y}_{\mathrm{n}}$ fixed and letting $\mathrm{A}$ run through the Borel sets, $\tilde{\mu}_{E}\left\{x \in E:\left(\left\langle x, y_{1}\right\rangle, \ldots,\left\langle x, y_{n}\right\rangle\right) \in{ }^{*} A\right\}=\mu_{F}\left\{x \in F\left\{\left\langle x, y_{1}\right\rangle, \ldots,\left\langle x, y_{n}\right\rangle\right) \in A\right\}$ (where $F$ is the subspace of $H$ generated by $y_{1}, \ldots, y_{n}$ ) defines a Radon measure on $\mathbb{R}^{n}$. Given an $\varepsilon>0$, there is thus an open set $G \supset A$, and a compact set $C \subset A$, such that

$\mu_{E}\left\{x \in E:\left(\left\langle x, y_{1}\right\rangle, \ldots,\left\langle x, y_{n}\right\rangle\right) \in{ }^{*} \mathrm{C}\right\}+\varepsilon \geq \mu_{E}\left\{x \in E:\left(\left\langle x, y_{1}\right\rangle,,_{\infty},\left\langle x, y_{n}\right\rangle\right) \in{ }^{*} A\right\}$ $\geq \mu_{E}\left\{x \in E:\left(\left\langle x, y_{1}\right\rangle, \ldots,\left\langle x, y_{n}\right\rangle\right) \in{ }^{*} G\right\}-\varepsilon_{0}$ Now ${ }^{*} \mathrm{C} \subset \mathrm{st}{ }^{-1}(\mathrm{C})$ and ${ }^{*} \mathrm{G} \supset \mathrm{st}^{-1}(\mathrm{G})$, and hence 
$\left\{\mathrm{x} \in \mathrm{E}:\left(\left\langle\mathrm{x}, \mathrm{y}_{1}\right\rangle, \ldots,\left\langle\mathrm{x}, \mathrm{y}_{\mathrm{n}}\right\rangle\right) \in{ }^{*} \mathrm{C}\right\} \subseteq\left\{\mathrm{x} \in \mathrm{E}:\left(\left\langle\mathrm{x}, \mathrm{y}_{1}\right\rangle, \ldots,\left\langle\mathrm{x}, \mathrm{y}_{\mathrm{n}}\right\rangle\right) \in \mathrm{st}^{-1}(\mathrm{~A})\right\} \subseteq$ $\subseteq\left\{\mathrm{x} \in \mathrm{E}:\left(\left\langle\mathrm{x}, \mathrm{y}_{1}\right\rangle, \ldots,\left\langle\mathrm{x}, \mathrm{y}_{\mathrm{n}}\right\rangle\right) \in^{*} \mathrm{G}\right\}$

Since $\epsilon$ is arbitrary, this implies that $\nu$ and $\tilde{\mu}$ agree on the cylinder sets, and the theorem is proved.

The theorem above is more general than the versions in e.g. Gross [5], or Kuo [10] as they treat only the case where $\mu$ is normally distributed. I have found no references to the actuel theorem in the literature, but it certainly ought to be wellknown. For applications the reader should consult Kuo [10].

The pattern of the proof is again the same; a limit measure exists naturally on an infinite element $E$, and using a suitable standard part map it is pushed down to a standard space. That we have been able to prove three central theorems in the theory of limit measures using this simple idea, should indicate that the Loeb-measure approach is both natural and promising; and I certainly hope that some original research may be carried on along these lines. Promising subjects should include stochastic integration in Hilbert spaces, and partial stochastic differential equations. 


\section{References}

1. R.M. Anderson: A Non-standard Representation of Brownian Motion and Itô Integration, Israel J. Math. 25(1976) pp. 15-46.

2. R.M. Anderson: Star-finite Probability Theory, Ph.D.-thesis, Yale University, 1977.

3. R.M. Anderson and S. Rashid: A Nonstandard Characterization of Weak Convergence, Proc. Amer. Math. Soc. 69 (1978) pp. 327-332.

4. P. Billingsley: Convergence of Probability Measures, John Wiley and Sons, 1968.

5. I. Gross: Abstract Wiener Spaces, Proc。5th. Berkeley Sym.Math. Stat. Prob. 2 (1965), pp. 31-42.

6. I.I. Helms: A Nonstandard Approach to the Martingale Problem for Spin Models, Preprint, University of Illinois, 1979.

7. I.I. Helms and P.A. Loeb: Applications of Nonstandard Analysis to Spin Models, J. Math. Anal. Appl。 69 (1979) pp. 341-352.

8. C.W. Henson: Analytic Sets, Baire Sets and the Standard Part Map, Can。 J. Math. XXXXI (1979) pp. 663-672.

9. A.E. Hurd: Nonstandard Analysis and Lattice Statistical Mechanics: A Variational Principle. Preprint, University of Victoria, 1977.

10. H. - H. Kuo: Gaussian Measures in Banach Spaces, INM 463, Springer-Verlag 1975.

11. P.A. Loeb: Conversion from Non-standard to Standard Measure Spaces and Applications in Probability Theory, Trans. Amer. Math. Soc., 211 (1975), pp. 113-122。 
12. P.A. Loeb:

13. P.A. Loeb:

14. Yu.V. Prohorov:

15. M. Reed and B. Simon: Methods of Modern Mathematical Physics I, Academic Press, 1972.

16. V.V. Sazonov: A Remark on Characteristic Functionals, Teor。Veroj。i Prim。3 (1958), pp.201-205.

17. I. Schwartz: Radon Measures on Arbitrary Topological Spaces and Cylindrical Measures, Oxford University Press, 1973.

18. K.D. Stroyan and W.A.J. Luxemburg: Introduction to the Theory of Infinitesimals. Academic Press, 1976. 